\title{
A radioactive source of exponentially distributed interevent intervals with electronically controlled rate and origin parameters
}

\begin{abstract}
JAMES N. CRONHOLM, EXPERIMENTAL PSYCHOLOGY DIVISION, U.S. ARMY MEDICAL RESEARCH LABORATORY, Fort Knox, Kentucky 40121
\end{abstract}

The equipment described utilizes the fact that time intervals between successive disintegrations in an appropriately chosen radioactive source are exponentially distributed. $A$ source and detector drive a probability unit that reduces the input distribution-rate parameter by as much as two decades. The origin parameter is then introduced by a gating circuit that eliminates pulses that follow their predecessors by less than a specified number of seconds. The system is reliable, and easily calibrated and adjusted.

Recent studies of reaction time (Nickerson \& Burnham, 1969), signal detection (Green \& Luce, 1967), reinforcement schedules (Millenson, 1963), and expectancy (Nickerson, 1967) have required the generation of sequences of interevent intervals that are approximately exponentially distributed. A unique property of this continuous distribution makes it especially interesting in such settings: waiting time to the next event is independent of the time already elapsed since the last event (McGill, 1963).

The exponential distribution has the form:

$$
f(t)=\mathrm{re}^{-\mathrm{r}(\mathrm{t}-\tau)}, 0 \leqslant \tau \leqslant \mathrm{t}, 0<\mathrm{r},
$$

in which $\tau$ is the smallest permissible interevent interval (in seconds) and $r$ is the rate parameter (in events per second). The mean of the distribution is $\tau+1 / \mathrm{r}$, which provides a simple estimate of $r$ when $\tau$ is known.

While there are several ways of generating exponential temporal distributions, the most frequently used system includes a radioactive source and a radiation detector mounted on a movable carriage. By changing the distance between the source and detector, the parameter $r$ can be varied (the origin parameter $\tau$ being zero). This is a rather complex arrangement, requiring precise mechanical alignment and separate lead shields for the source and detector. This note describes a source of exponential interevent intervals that is easily calibrated and adjusted but has no moving parts.

Figure 1 shows a block diagram of the device. A source of beta radiation is supplied by a small patch of carbon 14 painted on a stainless steel disk. This disk is mounted directly over the mica window of a Geiger-Muller tube, the whole assembly being shielded from ambient radiation by 2 in. of lead. The output of the counter (US Navy AN/PDR-27J Radiac set) is led to a 20-dB amplifier (H. H. Scott, Model 140B). Positive-going pulses from the amplifier are next clipped at $+10 \mathrm{~V}$ and the baseline clamped to $-10 \mathrm{~V}$ (see Time Line A in Fig. 1). In this form, the pulses are suitable for driving a BRS Precision Probability Unit (Model No. PP-1). The probability unit presents a constant, but controllable, transmission probability to pulses arriving at its input terminals; i.e., a pulse arriving at its input has a probability, $p$, of appearing simultaneously at its output, where $p$ is selected by front panel controls in increments of .01 .

Before proceeding, it will be shown that if the intervals of time separating input pulses to the probability unit are exponentially distributed with rate parameter $\beta$, then the distribution of
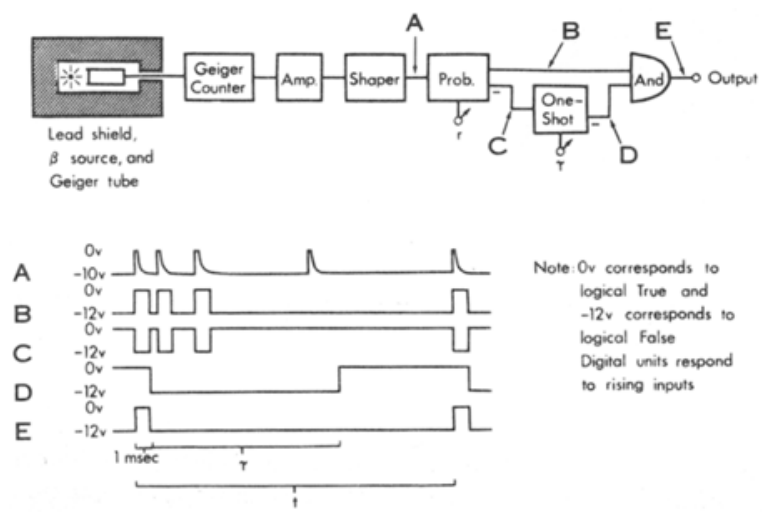

Fig. 1. Block diagram of the equipment. Waveforms at Points $A$ through $E$ are also shown.

interpulse intervals at its output will also be exponential, but with rate parameter $\mathrm{p} \beta$. On the one hand, if a pulse has just appeared at the output, the probability that the next $n-1$ input pulses will be rejected while the nth pulse is transmitted is given by the geometric distribution:

$$
u(n)=p q^{n-1}, n=1,2,3, \ldots, q=1-p .
$$

On the other hand, the time between the initially transmitted pulse and the nth input pulse following it is the sum of $n$ independent interpulse intervals, each of which is distributed exponentially with rate parameter $\beta$. The probability that the total interval is $\mathrm{t} \sec$ in duration is thus given by the $\mathrm{n}$-fold convolution of the exponential distribution with itself. This convolution is the gamma distribution with scale parameter $\beta$ and shape parameter n (MoGill, 1963, p. 320):

$$
v(t \mid n)=\frac{\beta^{n}}{(n-1) !} t^{n-1} e^{-\beta t}
$$

The joint density of $t$ and $n$ is the product of $u(n)$ and $v(t \mid n)$ :

$$
w(t, n)=p \beta e^{-\beta t} \frac{1}{(n-1) !}(q \beta t)^{n-1} .
$$

Summing this over all values of $n$ gives the required distribution:

$$
\begin{aligned}
f(t) & =p \beta e^{-\beta t} \sum_{n=1}^{\infty} \frac{1}{(n-1) !}(q \beta t)^{n-1} \\
& =p \beta e^{-\beta t} e^{q \beta t} \\
& =p \beta e^{-p \beta t} .
\end{aligned}
$$

The parameter $\beta$ is a constant of the radioactive source and the detector $\left(\beta=.556 \mathrm{sec}^{-1}\right.$ in the equipment described here), but the product $r=p \beta$ can be varied between 0 and $\beta$ simply by changing $\mathrm{p}$ on the calibrated controls of the probability unit. 


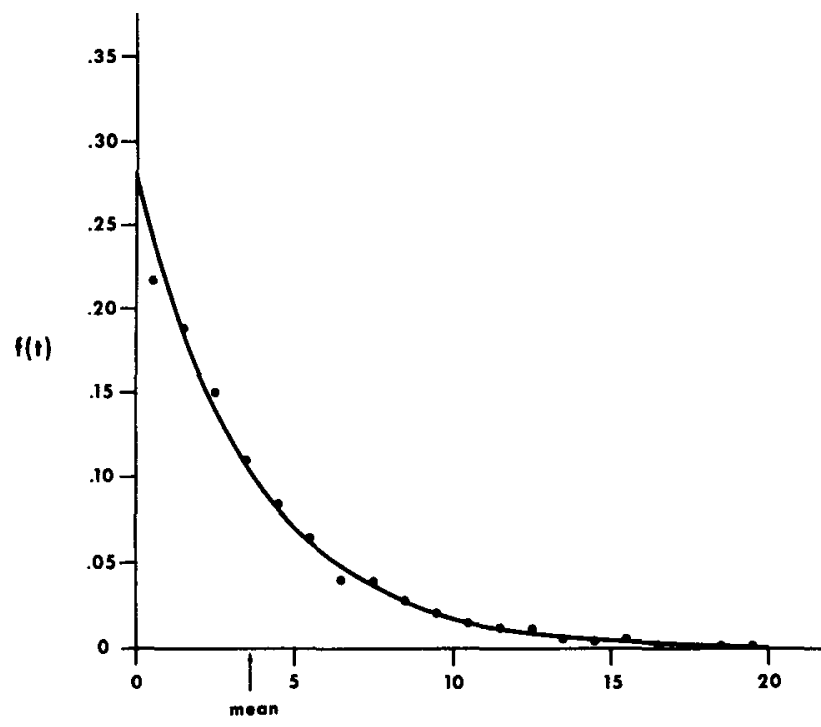

t(sec.)

Fig. 2. An observed distribution of 4,676 interevent intervals obtained during 36 7.5-min periods. The recording equipment did not register interevent intervals shorter than .1 sec, which accounts for the low observed value of the first point. Controls were set at $p=.50, \tau=0 \mathrm{sec} ; \beta=.556 \mathrm{sec}^{-1}, r=p \beta=.278 \mathrm{sec}^{-1}$, or 1,000 events per hour. By setting $p$ between 1 and .01 , the mean rate could be varied between 2,002 and 20 events per hour. The smooth curve was obtained from the integral of Eq. 1 with $r=.278 \mathrm{sec}^{-1}$ and $\tau=0$. The linear least-squares estimate of the hazard function for these data, i.e., the probability of an event in an interval $\Delta t=1 \mathrm{sec}$ at time $t$, given no event prior to time $t$, has a slope of -.0001 and an intercept of .283 . This illustrates the essential memoryless character of the exponential distribution.

A further word about the probability unit is in order. The device used is actually a temporal window with a variable aperture and a natural period of $10 \mathrm{msec}$. The probability controls determine the proportion of each successive $10-\mathrm{msec}$ period during which input pulses are passed to the output circuit. The output circuit reshapes these to form pulses of about 1-msec duration (see Time Lines B and C in Fig. 1).

These characteristics suggest two points. First, the periodic nature of the probability unit implies that the resulting distribution is very nearly geometric with an epoch duration of 10 msec. It is not exactly geometric since more than one pulse may be passed during the on portion of each cycle, the exact number depending on $p$ and the output pulse width. In most practical cases, however, a 10-msec epoch duration is very small compared to the mean interevent interval and Eq. 1 will provide an excellent fit (see Fig. 2).

A second limitation imposed by the periodic nature of the probability unit should be mentioned. One might be tempted to drive a second probability unit with the exponential output of the first in order to obtain even lower mean rates. This will not work, however, because the exponential output of the first unit is periodic in the sense described, and the second unit would have about the same period. As the two probability units go in and out of phase, the second unit will alternately pass and block all pulses from the first. Better results can be obtained by shielding the detector from the radioactive source or by increasing its distance from the source. In the former case, the shield should be placed over the detector window (rather than near the source) to avoid scattering effects that could influence the distribution form (Overman \& Clark, 1960, p. 214).

Returning to Fig. 1, the normal output (positive-going pulses) of the probability unit is connected to one input leg of an AND-gate (BRS, Model No. AG-102), while the inverted output drives a variable one-shot multivibrator (BRS, Model No. OS-102). The inverted output of the one-shot is connected to the other input leg of the AND-gate. This part of the circuit serves to introduce the origin parameter of Eq. 1 by blocking pulses that follow their predecessors by less than a fixed time, $\tau$, determined by the duration control on the one-shot.

When a pulse occurs and the one-shot is in its stable state, it is passed by the AND-gate and appears at the output (see Time Lines B, D, and E of Fig. 1). The inverted /trailing edge of this pulse also triggers the one-shot which closes the AND-gate to following pulses as long as it is in its unstable state, i.e., $\tau$ sec. When the one-shot reverts to its stable state, the next pulse to arrive will be passed and the process repeats.

A simpler, but less flexible, means of introducing the origin parameter involves connecting an external capacitor to the terminals provided on the probability unit. This increases the output pulse width by about $5 \mathrm{msec} / \mathrm{mF}$. Since each pulse terminates before another can be produced, the desired effect is achieved. Values of $\tau$ up to several seconds can be obtained in this way.

Whichever method is used, the distribution of time-interval durations between leading (or trailing) edges will have the form specified by Eq. 1: The probability unit controls determine the rate parameter $\mathrm{r}$ and the one-shot control determines the origin parameter $\tau$.

Several joint criteria should be considered when choosing a radionuclide. The isotope or labeled compound should be safe, inexpensive, available without AEC license, have a long half-life, and ideally, a single decay mode to a stable form. In the small amounts required, the safety, expense, and licensing requirements are readily met. Suppose, for example, that the system is to produce exponential distributions with mean interevent intervals ranging from 1 to $100 \sec (\tau=0)$. Assuming that one out of four disintegrations is detected, as little as approximately .1 nanocurie will suffice $\left(1\right.$ curie $=3.7 \times 10^{10}$ disintegrations per second). No AEC or state license is ordinarily needed to obtain nuclides in this amount.

Of the three basic kinds of radiation emitted ( $\alpha, \beta$, and $\gamma$ ), beta particles are a good choice because they are generally easier to detect than alpha particles, and easier to shield than gamma rays. There are six pure beta emitters with half-lives of 10 or more years (y) that decay to stable isotopes: hydrogen 3 (tritium), $12.26 \mathrm{y}$; beryllium $10,2.7 \times 10^{6} \mathrm{y}$; carbon $14,5770 \mathrm{y}$; argon 39 , $260 \mathrm{y}$; nickel 63, $92 \mathrm{y}$; and thenium $187,7 \times 10^{10} \mathrm{y}$ (Weast, 1965). Of these, carbon, hydrogen, and nickel appear to be most readily available. Mixtures of active isotopes should be avoided because the distribution of times between disintegrations may not be exponential. Similarly, parent nuclides that decay to active daughter isotopes have initially nonexponential decay curves, though they may be used after an appropriate period of aging (Overman \& Clark, 1960, Chap 8).

\section{REFERENCES}

GREEN, D. M., \& LUCE, R. D. Detection of auditory signals presented at random times. Perception \& Psychophysics, 1967, 2, 441-450.

McGILL, W. V. Stochastic latency mechanisms. In R. D. Luce, R. R. Bush, \& E. Galanter (Eds.), Handbook of mathematical psychology. Vol. 1. New York: Wiley, 1963.

MILLENSON, V. R. Random interval schedules of reinforcement. Joumal of the Experimental Analysis of Behavior, 1963, 6, 437-443.

NICKERSON, R. S. Expectancy, waiting time, and the psychological refractory period. Acta Psychologica, 1967, 27, 23-34.

NICKERSON, R. S., \& BURNHAM, D. W. Response times with nonaging foreperiods. Journal of Experimental Psychology, 1969, 79, 452-457.

OVERMAN, R. T., \& CLARK, H. M. Radioisotope techniques. New York: McGraw-Hill, 1960.

WEAST, R. C. (Ed.), Handbook of chemistry and physics. (46th ed.) Cleveland: Chemical Rubber Publishing Co., 1965. 